\title{
Application of analytic hierarchy process in international trade practice: research on supplier selection evaluation system based on M company
}

\author{
Lulu Yu ${ }^{1, a,{ }^{*}}$ \\ ${ }^{1}$ School of Economics and Management of Northwestern Polytechnical University Mingde \\ College,Xi 'an, shaanxi, China \\ aRainbow_yll@163.com,
}

Keywords: Analytic hierarchy process, Supplier selection, Selection evaluation system

\begin{abstract}
In this paper, the application of AHP in international trade practice is discussed from the perspective of supplier selection evaluation of $\mathrm{M}$ company by using international trade practice data and expert scoring.The results show that the establishment of AHP model and the final index screening based on the target layer can provide quantifiable reference data for import and export enterprises.
\end{abstract}

\section{层次分析法在国际贸易实务中的应用：基于M公司的供应商选择评价 体系研究}

\author{
余露露 $1, a,{ }^{*}$ \\ 1 西北工业大学明德学院经济与管理学院, 西安, 陕西, 中国 \\ aRainbow_yll@163.com
}

关键词:层次分析法，供应商选择，选择评价体系

中文摘要. 本文从 $\mathrm{M}$ 公司的供应商选择评价层面入手, 采用国际贸易实操数据和专家打分, 探讨 层次分析法在国际贸易实务中的应用。结果表明：层次分析法模型建立，基于目标层的最终指 标篮选，能够为进出口企业提供可量化的参考数据。

1. 引言

根据供应链的6+1体系理论可知，国际贸易实务的开展离不开供应商的严谨篮选，尤其是 为了满足不同地域市场的目标客户需求，减少企业的订单分配权衡时间，为之提供科学的评 价模型就显得尤为重要。

M公司立足于石材行业的国际进出口业务，避免生产能力下降，交货不及时、石材质量达不 到市场需求等情况的发生, 针对目标市场的需求和公司自身运营模式, 在参考了供应商删选方面 的参考文献过后，决定建立层次分析法理论（AHP），因为此理论是定量与定性的研究，并且有 科学的模型，整体模型的供应商选择方法易上手而且结果分别。具体模型的开展工作首先是确立 目标层--最优供应商的选择，在此目标层下建立业绩评估、生产能力评估、质量系统评估和发展 潜力评估四个准则层, 并针对每个准则层下设对供应商要求的指标层, 最后的方案层就是三家备 选择的供应商。 


\section{2. 理论机制}

层次分析法是旨在为解决一个核心目标问题而建立的模型，对于选择最优供应商的这个目 标，实际的操作步骤有以下五步:

（1）明确要解决的核心问题一一目标层：为核心企业选择最优的产品供应商。

（2）建立层次结构: 根据一定的评价指标为企业建立层次指标，其中包括目标层、准则 层、指标层和方案层。

（3）建造判断矩阵：对每一层相对于上一层的重要性做两两判断比较，并给出适度的判 断。

（4）层次单排序：每一层针对目标供应商有相应指标的评价值，构成判断矩阵，并计算出 相应权重值。方法是计算矩阵A的满足等式AW $=\lambda_{\mathrm{W}}$ 的最大特征根 $\lambda_{\text {max 和相对应的特征向量 }}$ $\mathrm{W}$ ，这个W就是单排序权值。

（5）层次总排序：计算每个候选供应商指标的总得分，根据建立的模型权重，计算总排 序，此排序就作为选择供应商的依据。

需要说明的是, 在进行层次单排序和层次总排序时都需要做一致性检验。所谓一致性检验是 使得整个的判断矩阵重要性排序具有一定的逻辑规格, 比如 $\mathrm{A}$ 的重要性大于 $\mathrm{B}, \mathrm{B}$ 的重要性大于 $\mathrm{C}$, 那么从逻辑上讲, $\mathrm{A}$ 的重要性就也应该大于 $\mathrm{C}$ 。但是如果出现了 $\mathrm{A}$ 的重要性不大于 $\mathrm{C}$, 那么就 存在逻辑不合理。所以, 一致性检验具有它的重要性, 它是整个模型权重排序正确性的保证。只 有一致性检验通过，才能说明判断矩阵在逻辑上是合理的，后续的结果分析才能继续。一致性检 验的步骤如下:

(1) 计算指标值C.I.。

$$
\text { C.I. }=\frac{\lambda_{\max }-\mathrm{n}}{\mathrm{n}-1}
$$

（2）查表值R.I，根据公式计算出C.R.

表1 随机一致性指标

\begin{tabular}{|c|c|c|c|c|c|c|c|c|c|c|c|c|c|}
\hline 阶数 & 3 & 4 & 5 & 6 & 7 & 8 & 9 & 10 & 11 & 12 & 13 & 14 & 15 \\
\hline R.I. & 0.58 & 0.89 & 1.12 & 1.24 & 1.32 & 1.41 & 1.45 & 1.49 & 1.52 & 1.54 & 1.56 & 1.58 & 1.59 \\
\hline
\end{tabular}

当C.R. $<0.1$ 时, 矩阵结果一致性, C.R. $>0.1$ 时, 矩阵不一致, 需要对该判断矩阵进行重新修 正。

\section{3. 层次分析法指标说明与模型设定}

\section{1 指标说明}

作为一家大型石材出口企业, M公司立足于与目标客户的长期合作发展, 在市场激烈的竞争 环境中，供应商的业绩、生产能力、质量以及未来发展潜力等因素显得尤为重要。旨在同等的质 量下提供最优的价格, 在相同的价格下提供优质的服务。所以, 业绩评估指标（价格因素）稍微 重要于生产能力指标, 也要比质量系统评估要重要但还不到相当主要的程度。

这里考虑到 $\mathrm{M}$ 公司属于加工出口企业，对于供应商的考核更加的关注于质量、交货、历史业 绩等因素, 而针对出口业务, 交货期的质量显得尤为突出, 当然产品多样性满足不同目标市场的 特殊偏好也需要考虑在内, 于是通过W公司管理层的考量, 基于多年的业务经验, W公司企业领 导、中层管理以及采购人员为了将定性的问题更加准确, 避免在决策建议上面产生漏洞, 进行了 鱼骨图分析法, 可以做到在对供应商选择的问题上面做到有条不紊、思路清晰。鱼骨图如下图: 


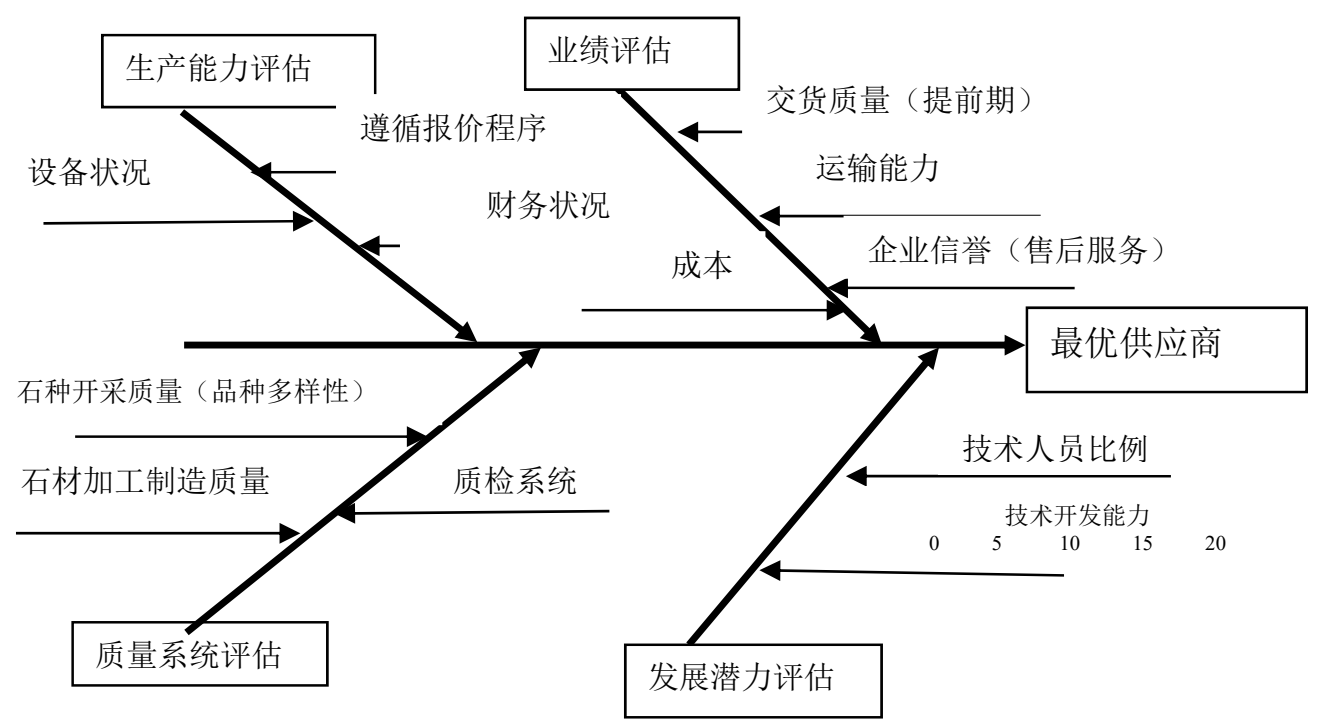

图1 M公司最优供应商鱼骨分析图

\section{2 层次结构模型设定}

在评价最优供应商的目标层下，将准则层列为四个部分：业绩评估、生产能力评估、质量系 统评估和发展潜力评估。在业绩评估准则层下含有成本、交货周期、运输能力和信誉四个指标; 在生产能力评估准则下有设备状况、遵循报价程序和财务状况三个指标; 在质量系统评估下有石 种开采质量、石材加工质量和质检系统三个指标; 在发展潜力评估准则下含有技术人员和技术开 发能力两个指标。最低层即为方案层, 是 $\mathrm{M}$ 公司在近年来的合作下要评定的三个供应商: 供应商 $\mathrm{A}$ 、供应商B和供应商C。

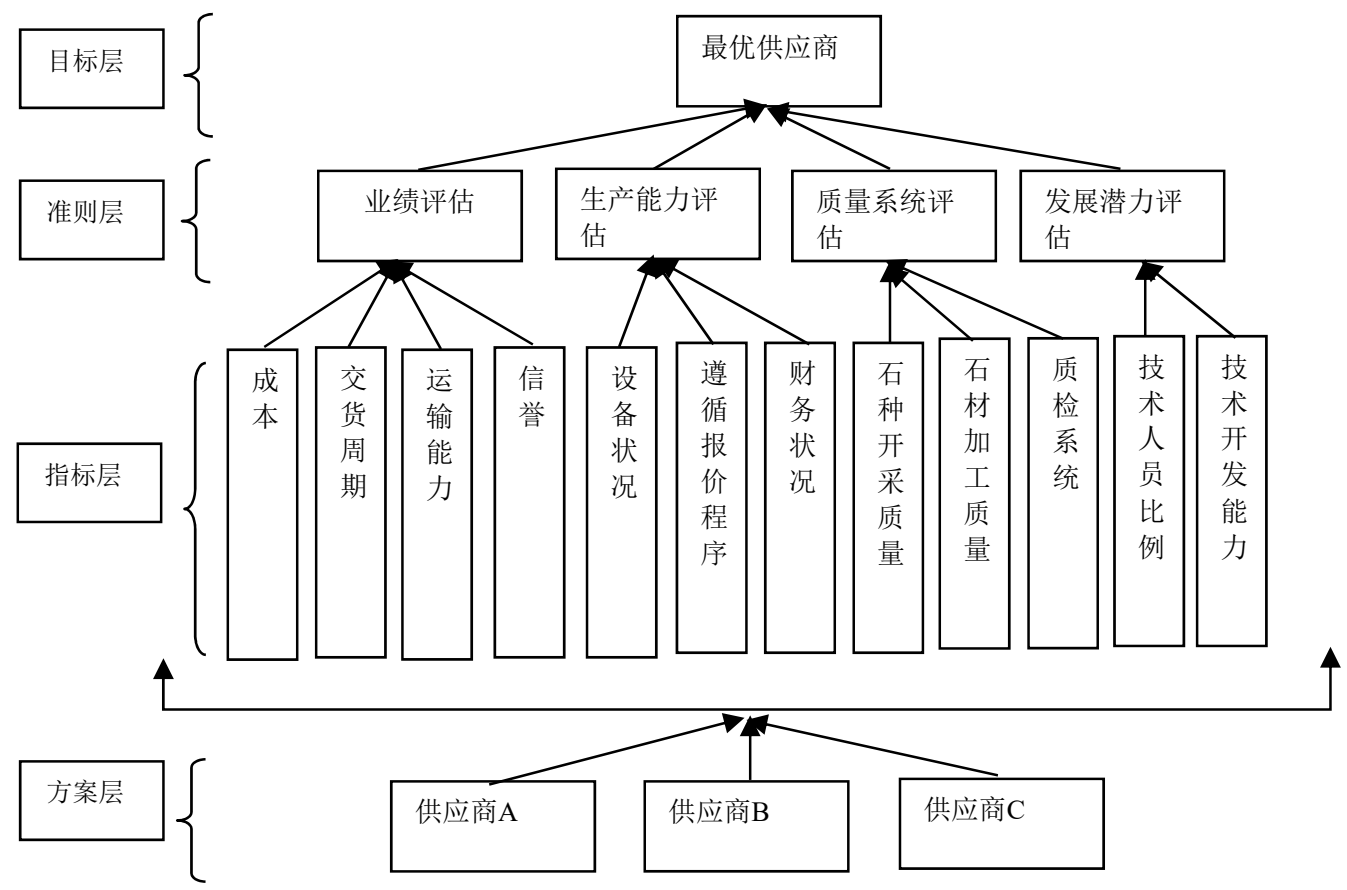

图2 层次结构模型

运用层次分析法框架罗列 $\mathrm{M}$ 公司选择最优供应商下四个准则包含的 12 个指标，如果此框架最 终运用层次分析法通过一致性检验的话，那么对于选择最优供应商的这个决策，此模型非常适 用。 


\subsection{1 建立层次分析法评价尺度表}

$\mathrm{M}$ 公司为了更加准确的把握准则层各个指标的相对重要性，面对中层管理人员、公司采购人 员以及公司重点培养的十个大客户设计调查问卷，共发放问卷数 10 份。针对问卷表格中管理人 员、采购人员以及客户的回答，将调查表格数据按照如下表层次分析法AHP评价尺度表转换成重 要性判断矩阵。

表2 AHP评价尺度

\begin{tabular}{|c|c|c|}
\hline 成对比较标准 & 定义 & 内容 \\
\hline & 同等重要 & \\
\hline 1 & (Equally Importance) & 两个要素具有同等的重要性 \\
\hline & 稍微重要 & 认为其中一个要素较另外一个要素稍微 \\
\hline 3 & $\begin{array}{l}\text { (Moderately More Importance) } \\
\text { 相当重要 }\end{array}$ & 重要 \\
\hline 5 & $\begin{array}{l}\text { (Strongly More Importance) } \\
\text { 明显重要 }\end{array}$ & 根据经验与判断, 强烈倾向于某一要素 \\
\hline 7 & (Very Strongly Importance) & 实际上非常倾向于某一要素 \\
\hline 9 & $\begin{array}{l}\text { 绝对重要 } \\
\text { (Extremely Importance) }\end{array}$ & $\begin{array}{l}\text { 有证据确定, 在两个要素比较时, 某一 } \\
\text { 要素非常重要, 即一个要素明显强于另 } \\
\text { 一个要素可控制的最大可能 }\end{array}$ \\
\hline 上述数值的倒数 & & $\begin{array}{l}\text { 用于上述标准之间的折中值 } \\
\text { 当甲要素与乙要素比较时, 若被赋予以 } \\
\text { 上某个标度值, 则乙要素与甲要素比较 } \\
\text { 时的权重就应该是那个标度的倒数 }\end{array}$ \\
\hline
\end{tabular}

表3 准则层对于目标层的对比矩阵

\begin{tabular}{ccccc}
\hline 供应商选择 & 业绩评估 & 生产能力评估 & 质量系统评估 & 发展潜力评估 \\
\hline 业绩评估 & 1 & 3 & 4 & 2 \\
生产能力评估 & $1 / 3$ & 1 & $1 / 2$ & $1 / 2$ \\
质量系统评估 & $1 / 4$ & 2 & 1 & 2 \\
发展潜力评估 & $1 / 2$ & 2 & $1 / 2$ & 1 \\
\hline
\end{tabular}

3.2.2 准则层对于目标层的判断矩阵及单排序和一致性检验

(1) 层次单排序

对 $\mathrm{M}$ 公司判断矩阵的元素首先按行相乘获得一新向量, 其次将新向量的每个分量开 $\mathrm{N}$ 次方 (获得几何平均值），最后将所得向量归一化即为权重向量。各指标的权重之和应为 1 。

表4 准则层各指标权重分配表

\begin{tabular}{cccccccc}
\hline 供应商选择 & $\begin{array}{c}\text { 业绩评 } \\
\text { 估 }\end{array}$ & $\begin{array}{c}\text { 生产能力 } \\
\text { 评估 }\end{array}$ & $\begin{array}{c}\text { 质量系统 } \\
\text { 评估 }\end{array}$ & $\begin{array}{c}\text { 发展潜力 } \\
\text { 评估 }\end{array}$ & $\begin{array}{c}\text { 按行相乘 } \\
\text { 开N次方 }\end{array}$ & $\begin{array}{c}\text { 权重Wi } \\
\text { (归一化) }\end{array}$ \\
\hline 业绩评估 & 1 & 3 & 4 & 2 & 24 & 2.213364 & 0.482 \\
生产能力评估 & $1 / 3$ & 1 & $1 / 2$ & $1 / 2$ & 0.083333 & 0.537285 & 0.117 \\
质量系统评估 & $1 / 4$ & 2 & 1 & 2 & 1 & 1 & 0.218 \\
发展潜力评估 & $1 / 2$ & 2 & $1 / 2$ & 1 & 0.5 & 0.840896 & 0.183 \\
\hline
\end{tabular}

由上我们即得特征向量 $\mathrm{W}=(0.482,0.117,0.218,0.183)^{T}$ ，可以看出M公司在准则层的看 重顺序是：业绩评估〉质量系统评估〉发展潜力评估〉生产能力评估。

随后将以上四个指标分别与各自权重Wi相乘并相加得到 $\mathrm{AWi}$ ，再将AWi除以Wi，随后 
将四个除得的值相加求算数平均数, 即为矩阵的最大特征根。

表 5 求矩阵的最大特征根

\begin{tabular}{ll}
\hline Awi & Awi/wi \\
\hline 2.071 & 4.295 \\
0.478 & 4.086 \\
0.939 & 4.310 \\
0.767 & 4.189 \\
\hline
\end{tabular}

则 $\lambda_{\text {max }}=\sum \frac{(A W) i}{n W i}=4.220$, 所以 $\mathrm{CI}=\frac{\lambda_{\text {max }}-\mathrm{n}}{\mathrm{n}-1}=0.0733$ 。

（2）一致性检验

这里需要用到随机一致性指标。

表6 随机一致性指标

\begin{tabular}{lccccccccccccc}
\hline 阶数 & 3 & 4 & 5 & 6 & 7 & 8 & 9 & 10 & 11 & 12 & 13 & 14 & 15 \\
\hline R.I. & 0.58 & 0.89 & 1.12 & 1.24 & 1.32 & 1.41 & 1.45 & 1.49 & 1.52 & 1.54 & 1.56 & 1.58 & 1.59 \\
\hline
\end{tabular}

查表得知 $n=4$ 时, $R I=0.89$, 所以:

$\mathrm{CR}=\frac{C I}{R I}=\frac{0.0733}{0.89}=0.082<0.10$ 认为矩阵的一致性通过。因此认为该判断矩阵具有可以接 受的满意一致性。

3.2.3 指标层对于准则层的判断矩阵及单排序和一致性检验

(1) 业绩评估下各指标相对重要性比较

1）调查问卷

同样面对中层管理人员、公司采购人员以及公司重点培养的十个大客户设计调查问卷，共 发放问卷数10份，下同。

2）重要性判断矩阵生成

表7 业绩评估下各指标权重分配表

\begin{tabular}{ccccccccc}
\hline 业绩评估 & 成本 & 交货质量 & 运输能力 & 信誉 & 按行相乘 & 开N次方 & 权重 & \\
\hline 成本 & 1 & $1 / 2$ & 3 & $1 / 2$ & $3 / 4$ & 0.931 & 0.200 & $\lambda \max =4.081$ \\
交货质量 & 2 & 1 & 4 & 2 & 16 & 2.000 & 0.430 & $\mathrm{CI}=0.027$ \\
运输能力 & $1 / 3$ & $1 / 4$ & 1 & $1 / 3$ & 0 & 0.408 & 0.088 & $\mathrm{CR}=0.030$ \\
信誉 & 2 & $1 / 2$ & 3 & 1 & 3 & 1.316 & 0.283 & \\
\hline
\end{tabular}

成本、交货质量、运输能力和信誉的相互重要性由上表显示，最大特征根 $\lambda \mathrm{max}=4.081$, 进行一致性检验, 得出 $\mathrm{CI}=0.027<0.1$, 一致性检验通过, 这个判断矩阵具有一致的满意性。

(2) 生产能力评估下各指标相对重要性比较

1) 调查问卷

2）重要性判断矩阵生成

表8 生产能力评估下各指标权重分配表

\begin{tabular}{cccccccc}
\hline $\begin{array}{c}\text { 生产能力 } \\
\text { 评估 }\end{array}$ & $\begin{array}{c}\text { 设备 } \\
\text { 状况 }\end{array}$ & 遵循报价程序 & 财务状况 & 按行相乘 & 开N次方 & 权重 & \\
\hline 设备状况 & 1 & 3 & 2 & 6 & 1.817 & 0.540 & $\lambda \max =3.009$ \\
遵循报价程序 & $1 / 3$ & 1 & $1 / 2$ & $1 / 6$ & 0.550 & 0.163 & $\mathrm{CI}=0.0045$ \\
财务状况 & $1 / 2$ & 2 & 1 & 1 & 1.000 & 0.297 & $\mathrm{CR}=0.008$ \\
\hline
\end{tabular}


设备状况、遵循报价程序和财务状况的相互重要性由上表显示, 最大特征根 $\lambda \mathrm{max}=3.009$, 进 行一致性检验, 得出 $\mathrm{CI}=0.008<0.1$, 一致性检验通过, 这个判断矩阵具有一致的满意性。

(3) 质量系统评估下各指标相对重要性比较

1）调查问卷

2）重要性判断矩阵生成

表9 质量系统评估下各指标权重分配表

\begin{tabular}{cccccccc}
\hline 质量系统评估 & $\begin{array}{c}\text { 石种开 } \\
\text { 采质量 }\end{array}$ & $\begin{array}{c}\text { 石材加工 } \\
\text { 制造质量 }\end{array}$ & 质检系统 & 按行相乘 & 开N次方 & 权重 & \\
\hline 石种开采质量 & 1 & $1 / 3$ & $1 / 2$ & $1 / 6$ & 0.550 & 0.163 & $\lambda \mathrm{max}^{2}=3.009$ \\
石材加工制造质量 & 3 & 1 & 2 & 6 & 1.817 & 0.540 & $\mathrm{CI}=0.0045$ \\
质检系统 & 2 & $1 / 2$ & 1 & 1 & 1.000 & 0.297 & $\mathrm{CR}=0.008$
\end{tabular}

石种开采质量、石材加工制造质量和质检系统的相互重要性由上表显示，最大特征根 $\lambda \max =3.009$, 进行一致性检验, 得出 $\mathrm{CI}=0.008<0.1$, 一致性检验通过, 这个判断矩阵具有一致的满 意性。

（4）发展潜力评估下各指标相对重要性比较

1) 调查问卷

2）重要性判断矩阵生成

表10 发展潜力评估下各指标权重分配表

\begin{tabular}{lcccccc}
\hline 发展潜力评估 & 技术人员比例 & 技术开发能力 & 按行相乘 & 开N次方 & 权重 & \\
\hline 技术人员比例 & 1 & $1 / 2$ & $1 / 2$ & 0.707 & 0.333 & $\lambda \max =2$ \\
技术开发能力 & 2 & 1 & 2 & 1.414 & 0.667 & $\mathrm{CI}=0$
\end{tabular}

技术人员比例和技术开发能力的相互重要性由上表显示, 最大特征根 $\lambda$ max $=2$, 进行一致性检 验，得出 $\mathrm{CI}=0<0.1 ， 一$ 致性检验通过，这个判断矩阵具有一致的满意性。

\subsection{4 指标层对于准则层的总排序}

通过将业绩评估与成本、交货质量、运输能力和信誉; 生产能力评估与设备状况、遵循报价 程序和财务状况; 质量系统评估与石种开采质量、石材加工制造质量和质检系统; 发展潜力评估 与技术人员比例和技术开发能力各指标因素的重要性比较, 各判断矩阵均具有满意的一致性, 汇 总各指标的相对重要性的判断矩阵计算结果如下表所示:

表11 指标层对于准则层的总排序表

\begin{tabular}{cccccc}
\hline & 业绩评估 & 生产能力评估 & 质量系统评估 & 发展潜力评估 & 总排序 \\
\hline & 0.482 & 0.117 & 0.218 & 0.183 & \\
成本 & 0.2 & & & 0.096 \\
交货周期 & 0.43 & & & 0.207 \\
运输能力 & 0.088 & & & 0.042 \\
信誉 & 0.283 & & 0.136 \\
设备状况 & & 0.54 & 0.063 \\
遵循报价程序 & & 0.163 & 0.019 \\
财务状况 & & 0.297 & 0.035 \\
\hline
\end{tabular}




\begin{tabular}{cccc}
\hline 石种开采质量 & 0.163 & 0.036 \\
石材加工制造质量 & 0.54 & 0.118 \\
质检系统 & 0.297 & & 0.065 \\
技术人员比例 & & 0.333 & 0.061 \\
技术开发能力 & & 0.667 & 0.122 \\
\hline
\end{tabular}

至此， $\mathrm{M}$ 公司基于层次分析法的供应商选择评价系统模型建立完毕。可以看出，在模型中的 12 个指标中， $\mathrm{M}$ 公司最看重的是业绩评估准则下交货周期的指标，其次是信誉指标，最不看重的 是生产能力评估下的遵循报价程序指标。

\section{4. 结论与展望}

本文对M公司原有的供应商选择评价体系做进一步的优化，结合其业务开展模式和加工型出 口行业的特点, 提出了可以采用层次分析法模型来选择和评价供应商, 从中可以看出, 层次分析 法供应商选择和评价模型不仅考虑到了供应商在业绩评估、生产质量评估、质量系统评估和发展 潜力评估四个指标, 而且充分考虑到每个指标下的子包含因素的权重比较, 可以最终自下而上的 形成了一套石材制造型出口企业的供应商综合选择评价体系。

随着国际经济形势的不断变化，石材作为资源型生产制造出口行业，在政府政策的管辖下， 很多的石材公司都选择缩水, 即将自有工厂的订单逐步分化给配合供应商, 多趋向于贸易发展和 管理。那么供应商的选择和评价体系就显得尤为的重要。希望本文的供应商选择和评价体系的研 究能为制造型出口企业的业务发展提供一定的启发, 尤其能为沿海加工型出口行业, 在面临长周 期的业务流程下，对供应商的选择部分起到一定的借鉴作用。

\section{References}

[1] Abraham Mendoza,José A. Ventura. Estimating freight rates in inventory replenishment and supplier selection decisions[J]. Logistics Research ,2009,1(3-4):20.

[2] Heung-Suk Hwang, Chiung Moon, Chun-Ling Chuang et al.Supplier Selection and Planning Model Using AHP[D]. Korea: Kainan University, Hanyang University,2005.

[3] John M.Gleason. The 4th annual international conference on industrical engineering theory:An Analytic Hierarchy Process Frocess Framework for technology acquisition decisions related to library information services[C].USA: Creighton University, 1999.

[4] A Model of a Localized Cross-Border E-Commerce[J] . Abbas Asosheh,Hadi ShahidiNejad,Hourieh Khodkari. iBusiness . 2012 (02)

[5] The impact of e-commerce on international trade and employment[J] . Nuray Terzi. Procedia Social and Behavioral Sciences . 2011

[6] The Drivers and Impediments for Online Cross-Border Trade in Goods in the EU. Martens B,Turlea G. Digital Economy Working Paper 1 . 2012

[7] Resolution of Cross-border E-business Disputes by Arbitration Tribunals on the Basis of Transnational Substantive Rules of Law and E-business Usages;The Emergence of the Lex informatica. Antonis Patrikios. 21st BILETA Conference Globalization and Harmonisation in Technology . 2006

[8] Formulating the Mission of Business Organization by Reference to the Economic Market Requirements[J] . Marius Costel Esi. Procedia Economics and Finance . 2015

[9] Service quality, customer satisfaction and loyalty in automobile repair services sector[J] . 
Ernest Emeka Izogo,Ike-Elechi Ogba. International Journal of Quality \& Reliability Management . 2015 (3)

[10] The value co-creation process as a determinant of customer satisfaction[J] . Manuela VegaVazquez,María ángeles Revilla-Camacho,Francisco J. Cossío-Silva. Management Decision . $2013(10)$

[11] The role of social interactions in building internal corporate brands: Implications for sustainability[J] . Christine Vallaster,Adam Lindgreen. Journal of World Business . 2013 (3)

[12] Creating 'value' beyond the point of production: branding, financialization and market capitalization. Hugh Willmott. Organization . 2010

[13]Regional Branding Measures in Japan:Efforts in12Major Prefectural and City Governments. Takafumi Ikuta,Kou Yukawa,Hiroshi Hamasaki. Place Branding and PublicDiplomacy . 2007

[14] The effect of the Internet on international trade[J] . Caroline $\mathrm{L}$ Freund,Diana Weinhold. Journal of International Economics . 2003 (1)

[15]Formulating the Mission of Business Organization by Reference to the Economic Market Requirements[J] . Marius Costel Esi. Procedia Economics and Finance . 2015

[16] Winning the last mile of E-Commerce. Haul Lee,Scungin Whang. . 2002

[17] Managing effective third party logistics relationships: what does it take?. Sandor Boyson,Thomas Corsi,Martin Dresner,et al. Journal of Business . 1999

[18] Product Difference and Intraindustry Trade. Lee.W Meknight,Joseh.P.Baley. Journal of Ec onomics . 2007 